\title{
Progress in deep-UV photoresists
}

\author{
P B SAHOO*, R VYAS, M WADHWA and S VERMA \\ Semiconductor Complex Limited, Industrial Area Phase-8, Mohali 160 059, India
}

\begin{abstract}
Higher resolution can be achieved in lithography by decreasing the wavelength of the exposure source. However, resist material and their processing are also important when we move to a shorter wavelength lithography technology. This paper reviews the recent development and challenges of deep-UV photoresists and their processing technology.
\end{abstract}

Keywords. Photoresists; DUV lithography; chemically amplified (CA) resist; top surface imaging.

\section{Introduction}

Lithographic processes have been leading the way to miniaturization in semiconductor technology. Any lithographic process is characterized by its power (resolution) to resolve the smallest dimension. Grossly, the minimum resolution achievable with a lithography tool is a function of the exposure wavelength and numerical aperture (NA) of the lens system. Higher resolution can be achieved by decreasing wavelength or by increasing NA. However, the most dominant approach to improve resolution is to use a shorter wavelength. The modern lithography started with the spectral line of $436 \mathrm{~nm}$ ( $g$-line) and later $365 \mathrm{~nm}$ ( $i$-line) obtained from mercury arc lamp. Presently, semiconductor devices are being produced with dimensions of the order of $180 \mathrm{~nm}$ dimensions. At these dimensions deep ultraviolet (DUV) lithography is used, which employs $248 \mathrm{~nm}$ wavelength obtained from $\mathrm{KrF}$ excimer laser. At some advanced semiconductor foundries ArF excimer laser (193 nm) is also used to print the features of $130 \mathrm{~nm}$. It is predicted that future devices with dimensions of the order of $100 \mathrm{~nm}$ will be produced using lithography systems based upon $F_{2}(157 \mathrm{~nm})$ source.

Photoresist is another key parameter for lithographic processes. Any photoresist must offer three fundamental properties viz. high transparency at the exposure wavelength, etch resistance during subsequent plasma processes and ability to undergo efficient photochemical transformations. However, secondary requirements include accessibility through reasonably simple synthesis schemes, high glass transition temperatures as compared to processing temperature, adhesion to a wide range of substrates, acceptable shelf storage lifetime, and minimum toxicological risk. Transition from one wavelength to another necessitates the change in photoresist chemistry in order to fulfil above-mentioned requirements. The near

\footnotetext{
*Author for correspondence
}

UV (436-365 nm) technology is dominated by diazonapthaquinone (DNQ)/novolac based resists. But their excessive unbleachable absorption and low sensitivity at DUV wavelengths make them unsuitable for use in DUV region. In order to circumvent this intrinsic sensitivity limitation and to increase resist sensitivity, concept of chemically amplified (CA) resists was introduced by researchers (Ito and Wilson 1982).

\section{Chemical amplification in resists}

In CA resists, an additional photoactive compound commonly called photo acid generator (PAG) is added to the polymer matrix and photosensitizer. The PAG produces a small amount of acid, when exposed to light by photochemical decomposition. During post-exposure bake (PEB), the acid activates a cascade of subsequent chemical transformations in the resists causing a polarity change in the polymer from lipophilic to hydrophilic, making exposed regions soluble in basic developers like tetra-methyl ammonium hydroxide (TMAH). Thus, the previously generated acid acts as a catalyzer during PEB and is hardly consumed by the reaction. This results in a dramatic increase in quantum efficiency and sensitivity of the exposure. CA resists need very low exposure doses as compared to conventional photoresists. The use of acid as a catalyst has allowed tremendous design versatility in CA resists (Ito 1997). In addition to enhanced sensitivity, high contrast and high resolution are the major reasons for wide acceptance of CA resists in industry. Imaging chemistry of CA resists is shown in figure 1.

The first commercial CA resist was negative tone resist based on cross-linking of a phenolic resin through acid catalyzed condensation (Ito 1997). This photoresist allowed the lithographic community to gain experience with CA resist and to witness its unexpectedly high-resolution capability. All negative resists available are built on this cross-linking of a phenolic resin. Negative DUV resists have not found wide use because they do not provide 


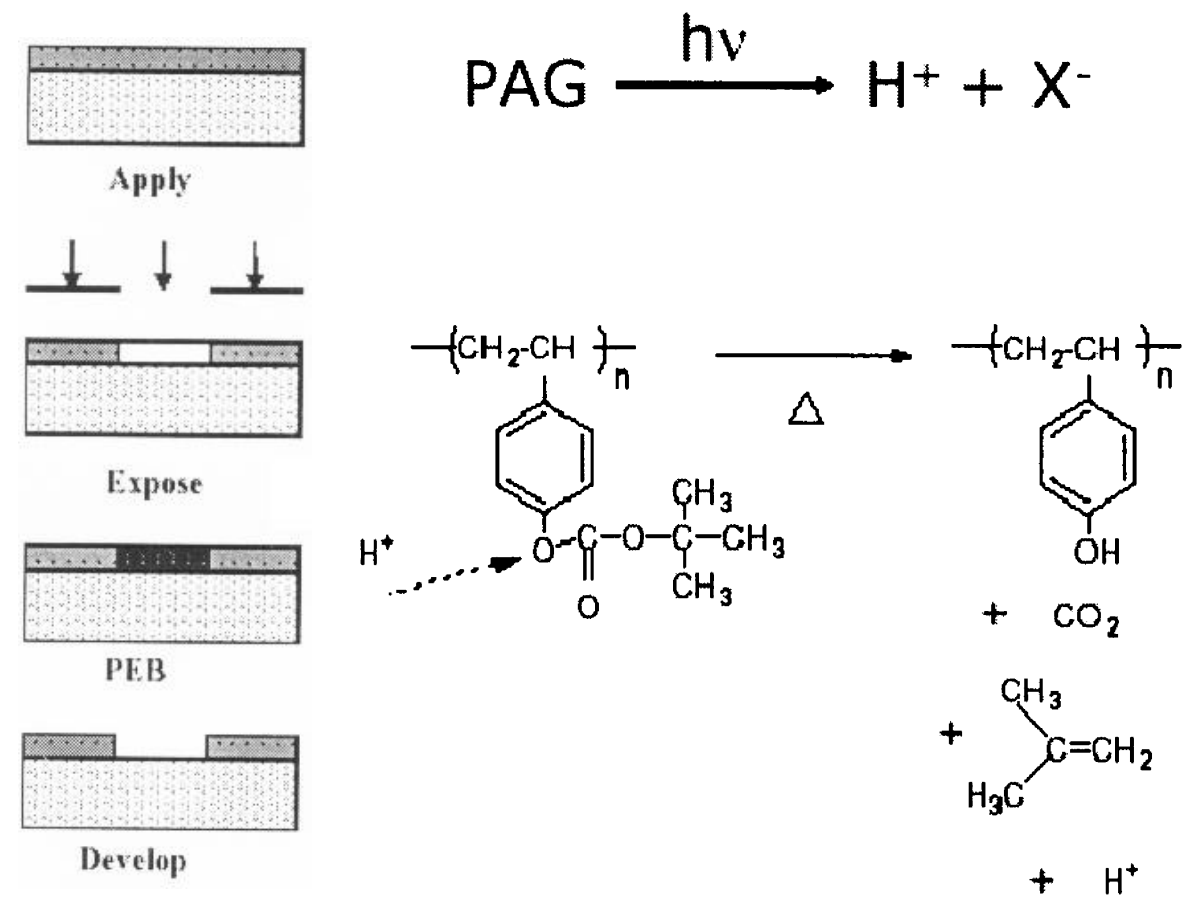

Figure 1. Imaging mechanism in chemically amplified resists.

good process latitude in imaging contact hole, due to the nature of the un-enhanced bright-field aerial image. Thus, positive CA DUV resists have attracted intensive development efforts for the last several years. All these positive resists are built on acid-catalyzed deprotection mechanism, converting a hydrophobic dissolution-inhibiting functionality to a hydrophilic base-soluble functionality. Presently $248 \mathrm{~nm}$ CA resist technology is in production and $193 \mathrm{~nm}$ CA resists technology is ready for production but $157 \mathrm{~nm}$ resist technology is still in its development phase.

\section{Challenges in chemically amplified photoresist}

Although CA resists demonstrate high sensitivity, high contrast and high resolution, they suffer from few common and serious problems viz. the appearance of an anomalous insoluble skin and linewidth shift when the PEB was delayed. Due to catalytic nature of the imaging mechanisms, CA resists are also susceptible to the presence of airborne base contamination and it is a yieldlimiting issue of modern resists (Kemp et al 1997). Elevated concentrations of molecular bases cause image degradation and reduce production output. It is a well established fact that ammonia and other volatile basic compounds can affect the performance of resists used to produce DUV images. The wafer is susceptible to molecular base (MB) contamination from the moment the resist is coated until the post-exposure bake step in the process. Pre-exposure basic contamination can cause

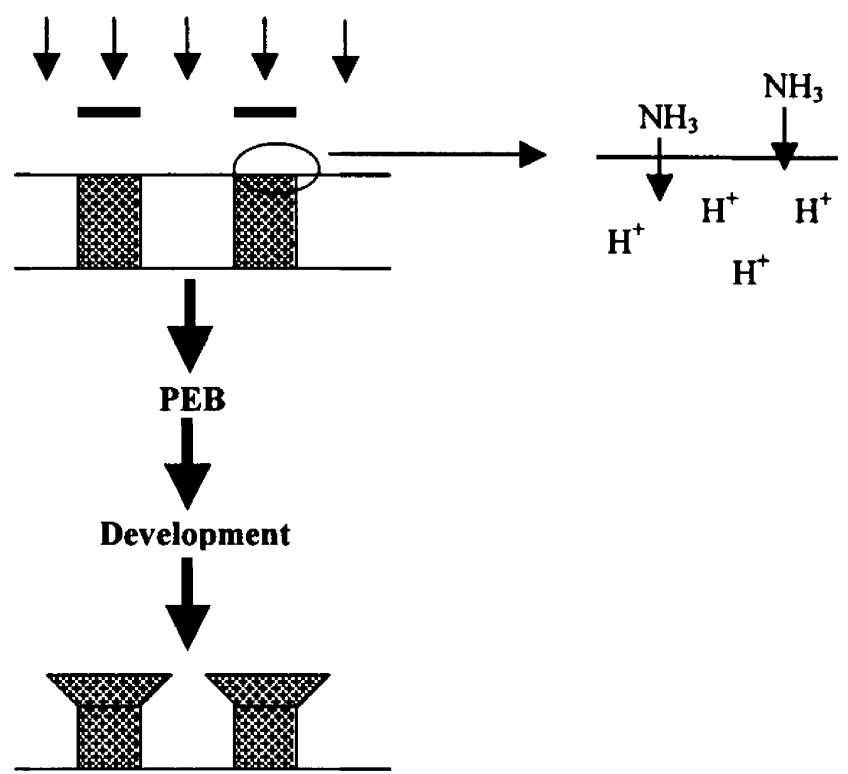

Figure 2. Indiffusion of basic contaminant through the top surface of the resist film and its impact showing T-topping on the resist profile.

typically a widening in the top of the image profile called a 'T-top'. Since exposure to environmental base contamination causes a change in the image profile, CA photoresists are said to be environmentally sensitive.

Figure 2 shows image degradation of CA resist due to contamination. This is why chemical filters are employed in DUV lithography equipment to control the effects 
of environmental base contamination, viz. T-topping. Although the matter of resist environmental sensitivity requires additional investigation, some $\mathrm{CA}$ resists may be affected by impurities in the air at very low levels down to 1 or 2 parts-per-billion (ppb). Other important challenges of CA resists are CD change due to acid diffusion if PEB is delayed and CD shrinkage during CDSEM.

\section{4. $248 \mathrm{~nm}$ and $193 \mathrm{~nm} \mathrm{CA}$ photoresists}

Presently $248 \mathrm{~nm}$ CA resist technology is in production and $193 \mathrm{~nm} \mathrm{CA}$ resist technology is ready for production. $248 \mathrm{~nm}$ CA resists are based on hydroxystyrene polymers, alone or in combination with other monomers such as $t$-butyl acrylate. Resist designers incorporate different PAGs, acid-labile deprotecting groups and additives to increase performance or tailor the resist for a specific application. Acrylic polymers are the new basis for $193 \mathrm{~nm}$ resists design because of their excellent optical transparency and easily tailored structure (Allen et al 1997). Acrylic polymers provide good resolution but lack in etch resistance. New polymers, called cyclo olefin maleic anhydrides (COMA) found improved etch resistance but lacked the needed resolution. This led to a dual approach that is still playing out, in which resist chemists improve etch resistance in acrylic resists without decrease in resolution, or enhance resolution in COMA resists without sacrificing etch resistance (Peters 2001). Recently several vendor-introduced $193 \mathrm{~nm}$ resists show large improvements in problem area such as line edge roundness and line slimming during CD SEM measurements.

\section{Development of $\mathbf{1 5 7} \mathbf{~ n m}$ photoresist materials}

Besides tremendous improvement in $193 \mathrm{~nm}$ resists technology, significant developments are going on with $157 \mathrm{~nm}$ CA resists to keep pace with ITRS roadmap. However, designing high-resolution photoresist for $157 \mathrm{~nm}$ poses a difficult challenge, since, air and most hydrocarbons are strongly absorbing at this wavelength. Current resist-platforms used for $248 \mathrm{~nm}$ and $193 \mathrm{~nm}$ exposure are also found to be too absorbing at reasonable thickness. Spectroscopic studies lead to the observation that highly fluorinated hydrocarbons and silicon based siloxanes offer the best hope for the transparency that is necessary for the design of an effective $157 \mathrm{~nm}$ photoresist, and these classes of materials have quickly become the prominent platforms for a variety of research activities in this field. The main challenge in developing a siloxane resist is achievement of a sufficiently high glass transition temperature.

For $157 \mathrm{~nm}$ CA resist technology major development is going on with modular approach (Patterson et al 2000a, b; Will et al 2001). The modular approach to the design of resist polymer requires identification of four 'modules': a backbone that provides basic mechanical properties, an etch barrier that provides reactive ion etching (RIE) resistance, an acidic group that generates tetra methyl ammonium hydroxide (TMAH) developer solubility, and an acid-labile protecting group that enables a chemical amplification type solubility switch. All these not only perform the specific functions, but also provide transparency at the $157 \mathrm{~nm}$ wavelength. Figure 3 shows modular approach for $157 \mathrm{~nm}$ photoresist materials.

\section{Top surface imaging (TSI)}

The TSI technique is a multilayer process. The use of additional layer allows one to circumvent many of the problems of single layer resist, but with increased cost. In these systems, the uppermost layer of the resist is imaged and this image is used to create an etch mask. Finally, the images are developed using an anisotropic RIE process. This type of imaging scheme has several benefits. Because only the top portion of the resist is imaged, the resist may be relatively opaque at the wavelength of the exposure tool, and a larger variety of materials may therefore be used as photoresists. Additionally, no light is reflected off the silicon substrate or existing topography on the wafer. Normally, this reflected light interferes with the light that is propagating through the resist to form standing wave patterns. It is undesirable to have standing waves in the resist because they make controlling the dimensions of the features difficult. By eliminating the aberrations that result from reflected light, the top surface imaging lithography system theoretically allows for better linewidth control. The next benefit of TSI processes concerns

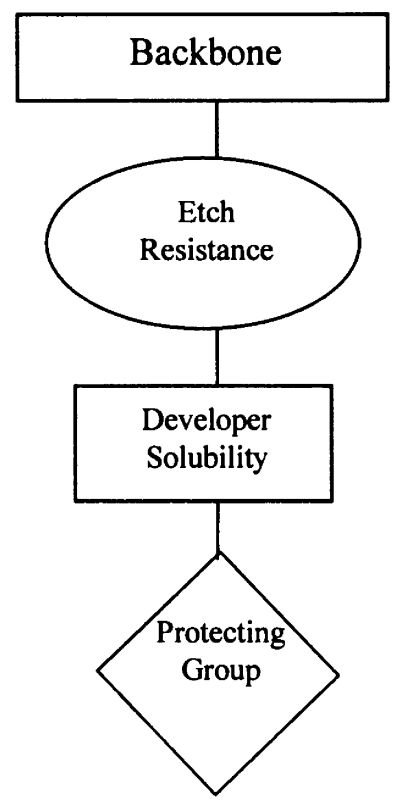

Figure 3. Modular approach to design $157 \mathrm{~nm}$ DUV photoresist. 

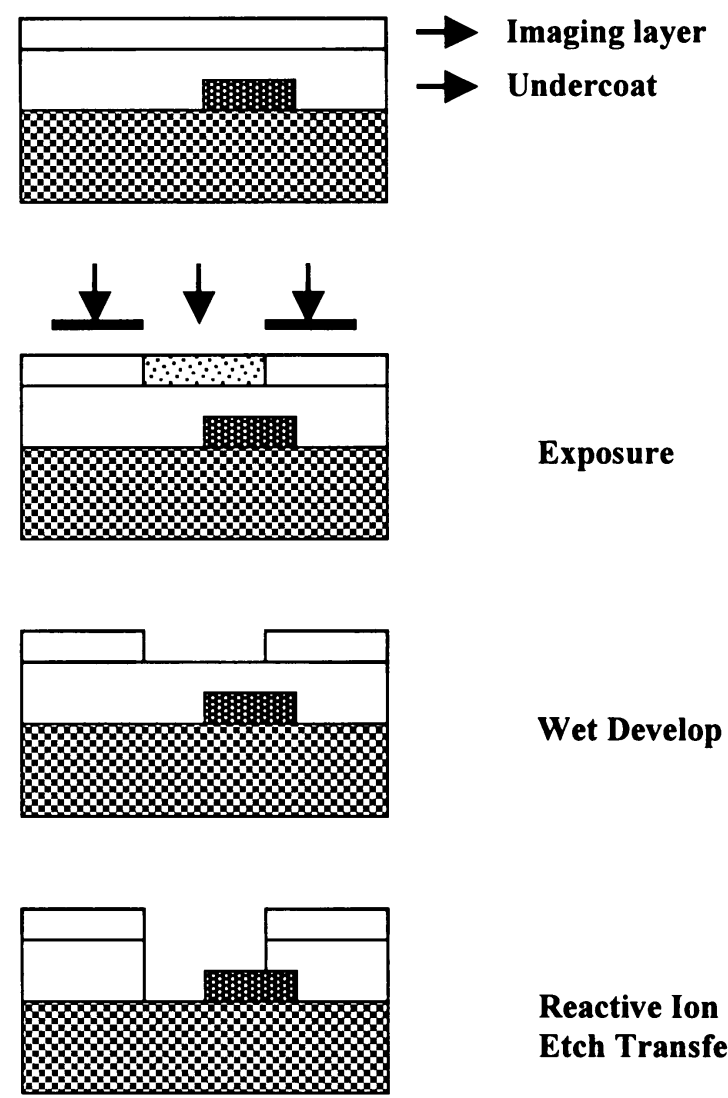

\section{Reactive Ion Etch Transfer}

Figure 4. Schematic of top surface imaging.

the 'depth of focus'. Microelectronic devices are made through the use of repeated lithography steps. As devices are built on the wafer, the wafer loses its planarization. The final benefit of the TSI system lies in its ability to produce high aspect ratio images. Despite all of these advantages, thin layer imaging techniques are used only when single layer technologies fail. Each of the TSI sys- tem has added process complexity that increases the cost of the manufactured devices. Conceptual design of TSI system is shown in figure 4 . The $248 \mathrm{~nm}$ resists are based on single layer resists technology but TSI technology is attractive for $193 \mathrm{~nm}$ and $157 \mathrm{~nm}$ resists family (Kunz et al 1999).

\section{Conclusion}

The chemically amplified resists exhibit high transparency, high contrast and high resolution in deep UV regions. Presently $248 \mathrm{~nm}$ CA resists are in production whereas $193 \mathrm{~nm}$ CA resists are ready for production. Besides $157 \mathrm{~nm}$ resist technology is still in its development phase. Materials and processes of CA resists will continue to support highly demanding future lithography technologies.

\section{Acknowledgements}

The authors would like to thank Mr Rajkumar and Mr V Malhan for their valuable advice and encouragement.

\section{References}

Allen R et al 1997 IBM J. Res. \& Devel. 4195

Ito H and Willson C G 1982 Technical paper of SPIE regional technical conference on photopolymers, Tokyo, Japan p. 331 Ito H 1997 IBM J. Res. \& Devel. 4169 Kunz R R et al 1999 Proc. SPIE 367813

Kemp K G et al 1997 SPIE Proc. 1295995

Patterson K et al 2000a Solid State Technol. 4341

Patterson K et al 2000b Proc. SPIE 3999365

Peters L 2001 Semicond. Int. 77

Will C et al 2001 Solid State Technol. 31 